\title{
Absolute vibration suppression (AVS) control - modeling, implementation and robustness
}

\author{
Y. Halevi* and I. Peled \\ Technion - Israel Institute of Technology, Haifa, Israel
}

\begin{abstract}
Absolute Vibration Suppression (AVS) is a control method for flexible structures governed by the wave equation. Such system may be a rotating shaft, a rod in tension or a crane for which the cable mass is not negligible. First an accurate, infinite dimension, transfer function, relating arbitrary actuation and measurement points, with general boundary conditions, is derived. The transfer function consists of time delays, due to the wave motion, and low order rational terms, which correspond to the reflection from the boundary. Thus the compact mathematical representation has a clear physical interpretation. Furthermore, it leads to the special structure of the AVS controller which is a collocated, rate to force feedback that completely eliminates the vibration. The paper investigates the properties of the AVS controller and its robustness to modeling errors, in particular small non-collocation.
\end{abstract}

Keywords: Control, wave equation, vibration suppression

\section{Introduction}

Control of flexible structures is one of the most challenging problems in control theory. Low damping, a large number of closely spaced resonance frequencies within the control bandwidth, and high performance requirements often make this problem extremely difficult. Standard model based control methods, such as pole placement or optimal control, result in controllers having a dimension at least equal to that of the model. The infinite, or very high, order of the entire model makes it impossible to use such methods, at least without order reduction. While some open loop methods, such as minimum time and input shaping [1] are useful in the control of flexible structures, most applications require closed loop control. Various approaches for closed loop control have been suggested, e.g. direct velocity feedback [2], uniform damping control [3], and applications of the general $\mathrm{L}_{2}$ and $\mathrm{H}_{\infty}$ optimization. Some of these methods apply only to regulation (vibration suppression), whereas others are applicable also to tracking as well [4].

The first step in the design of every control system is modeling. Flexible structures are governed by Partial Differential Equations (PDE), and hence have infinite dimension. However, most modeling methods use finite dimension approximations of the system. In the modal approach the infinite dimension appears as an infinite sum of spatial eigenfunctions multiplied by time functions. In practice though, only a finite sum is used. In the popular Finite Element Method (FEM), the finite dimension is achieved by spatial discretization. While finite approximation is practically and even conceptually convenient, some important properties of the system's behavior are lost by it.

A different approach was taken in [5,6] and is adopted in this paper. It considers the problem of deriving the accurate, infinite dimension, Laplace transfer function of the flexible system. The building blocks of this transfer function are time delays, representing the wave motion, and low order rational expressions, representing the boundary

\footnotetext{
*Corresponding author. E-mail: yoramh@technion.ac.il.
} 


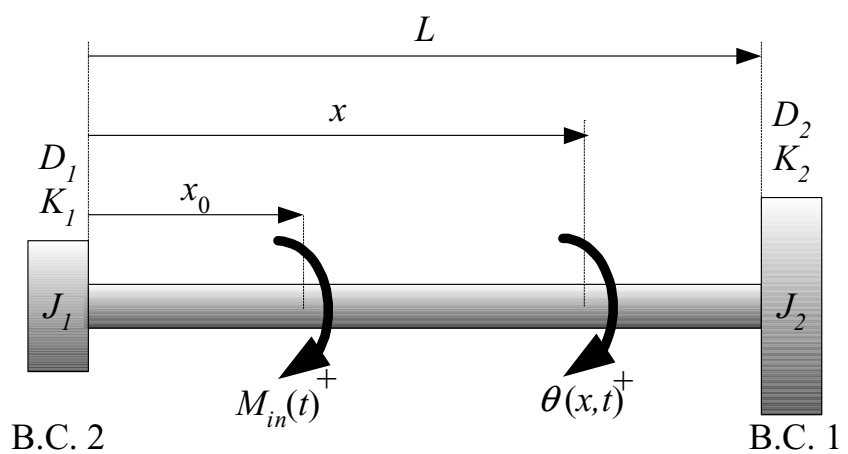

Fig. 1. The flexible system.

phenomena. The transfer function modeling approach has several theoretical and practical advantages, and it can be shown that most of the results from classical theory for conservative systems can be inferred from it. Furthermore, when the boundary conditions contain dampers, the results obtained via the transfer function do not have counterparts in classical modal analysis. The practical opportunities offered by this modeling approach include accurate yet simple simulation schemes, exact frequency response for the entire frequency range, analytical solution for the finite time response and identification of dedicated control laws, which is the subject of this paper. While transfer functions have been used before to model flexible structures, e.g. [7], neither one of the reported methods gives an explicit expression for general boundary conditions case, nor has clear physical interpretation.

Standard, model based, control strategies are difficult to apply to flexible structures mainly due to the very high dimension of the FEM or modal models, and the low damping in the system. In [5,8,9] the explicit, highly structured, and physically meaningful form of the transfer function was used to derive a closed loop control law, which was later termed Absolute Vibration Suppression (AVS). AVS is a collocated rate feedback with the unique property that it completely eliminates vibrations from the loop. In fact the closed loop transfer function becomes finite dimension plus the unavoidable delay. The delays in the transfer function correspond to the wave motion and from that point of view, the AVS performance is achieved by making the actuating end a sink for the returning wave. In [10] it is shown that the AVS controller bears similarities to wave based control methods [11-13]. The current paper is devoted to several stability and robustness issues associated with the AVS. In particular it considers the practical case of slight non-collocation of the controller.

\section{Infinite dimension transfer functions}

As an illustration of a system governed by the wave equation, consider the system in Fig. 1 showing a uniform rod of length $L$ subjected to a lumped torque moment $M(t)$ at the point $x=x_{0}$.

Assuming no internal damping, the torsion waves in the system are governed by the wave equation:

$$
\frac{1}{c^{2}} \frac{\partial^{2} \theta(x, t)}{\partial t^{2}}-\frac{\partial^{2} \theta(x, t)}{\partial x^{2}}=\frac{1}{G I_{p}} \cdot M_{i n}(t) \cdot \delta\left(x-x_{0}\right)
$$

where $\theta(x, t)$ is a torsion angle at distance $x$ from the left end, $I_{p}$ denotes the polar moment of inertia, $\rho$ is the material density, $G$ is the shear elasticity modulus, and $c=(G / \rho)^{1 / 2}$ is the wave propagation velocity. The boundary conditions are given by

$$
\begin{array}{ll}
I_{p} G \frac{\partial \theta(x, t)}{\partial x}=J_{1} \frac{\partial^{2} \theta(x, t)}{\partial t^{2}}+D_{1} \frac{\partial \theta(x, t)}{\partial t}+K_{1} \theta(x, t) & x=0 \\
I_{p} G \frac{\partial \theta(x, t)}{\partial x}=-\left(J_{2} \frac{\partial^{2} \theta(x, t)}{\partial t^{2}}+D_{2} \frac{\partial \theta(x, t)}{\partial t}+K_{2} \theta(x, t)\right) & x=L
\end{array}
$$


where $J_{i}, D_{i}$ and $K_{i}, i=1,2$ are the inertia, damper and spring constant at an end. This general setting includes all linear boundary conditions of interest. For example, in a free end all the coefficients are identically zero, and a fixed end is obtained when $K, D$, or $J \rightarrow \infty$.

A Laplace transform with respect to time converts the PDE Eq. (1) into an ODE in $x$ :

$$
\frac{\partial^{2} \theta(x, s)}{\partial x^{2}}-\frac{s^{2}}{c^{2}} \theta(x, s)=-\frac{1}{G I_{p}} \cdot M_{i n}(s) \delta\left(x-x_{0}\right)
$$

where $M_{i n}(s)$ and $\theta(x, s)$ denote Laplace transforms, with respect to time, of $M_{i n}(t)$ and $\theta(x, t)$ respectively. The boundary conditions become

$$
\begin{array}{ll}
I_{p} G \frac{\partial \theta(x, s)}{\partial x}=\left(J_{1} s^{2}+D_{1} s+K_{1}\right) \theta(x, s) & x=0 \\
I_{p} G \frac{\partial \theta(x, s)}{\partial x}=-\left(J_{2} s^{2}+D_{2} s+K_{2}\right) \theta(x, s) & x=L
\end{array}
$$

It is shown in [5] that the solution of Eq. (3) is

$$
\theta(x, s)=-\frac{c M_{i n}(s)}{4 G I_{p} s}\left(e^{\left(\frac{s\left|x-x_{0}\right|}{c}\right)}-e^{-\left(\frac{s\left|x-x_{0}\right|}{c}\right)}\right)+C_{1}(s) e^{\left(\frac{s x}{c}\right)}+C_{2}(s) e^{-\left(\frac{s x}{c}\right)}
$$

Substituting (5) into the boundary conditions (4) gives, after solving the two linear equations for $C_{1}(s)$ and $C_{2}(s)$, which in turn are linear in $M_{i n}(s)$ the following transfer function.

$$
\frac{\theta\left(x, x_{0}, s\right)}{M_{i n}(s)}=\left\{\begin{array}{l}
\frac{1}{2 \phi s} \cdot \frac{e^{-(\gamma-\delta) \tau s}+R_{1}(s) e^{-(\gamma+\delta) \tau s}+R_{2}(s) e^{-(2-\gamma-\delta) \tau s}+R_{1}(s) R_{2}(s) e^{-(2-\gamma+\delta) \tau s}}{1-R_{1}(s) R_{2}(s) e^{-2 \tau s}} \\
x \geqslant x_{0} \\
\frac{1}{2 \phi s} \cdot \frac{e^{-(\delta-\gamma) \tau s}+R_{1}(s) e^{-(\gamma+\delta) \tau s}+R_{2}(s) e^{-(2-\gamma-\delta) \tau s}+R_{1}(s) R_{2}(s) e^{-(2-\delta+\gamma) \tau s}}{1-R_{1}(s) R_{2}(s) e^{-2 \tau s}} \\
x \leqslant x_{0}
\end{array}\right.
$$

where

$$
\phi=\frac{G I_{p}}{c}, \quad \tau=\frac{L}{c}, \quad \gamma=\frac{x}{L}, \quad \delta=\frac{x_{0}}{L}
$$

and

$$
R_{i}(s)=\frac{\phi s-\left(J_{i} s^{2}+D_{i} s+K_{i}\right)}{\phi s+\left(J_{i} s^{2}+D_{i} s+K_{i}\right)} i=1,2
$$

The physical interpretation of these quantities will be discussed at the end of this section. By defining

$$
\beta=\frac{\left|x-x_{0}\right|}{L}, \quad \eta=\frac{\max \left(x, x_{0}\right)}{L},
$$

the two equations for the transfer function $G\left(x, x_{0}, s\right)$ (no relation with the elasticity modulus $G$ ) can be combined into the single expression.

$$
G\left(x, x_{0}, s\right)=\frac{1}{2 \phi s} \cdot \frac{e^{-\beta \tau s}+R_{1}(s) e^{-(2 \eta-\beta) \tau s}+R_{2}(s) e^{-(2-2 \eta+\beta) \tau s}+R_{1}(s) R_{2}(s) e^{-(2-\beta) \tau s}}{1-R_{1}(s) R_{2}(s) e^{-2 \tau s}}
$$

or equivalently

$$
G\left(x, x_{0}, s\right)=\frac{1}{2 \phi s} \cdot \frac{\left(1+R_{1}(s) e^{-2(\eta-\beta) \tau s}\right)\left(1+R_{2}(s) e^{-2(1-\eta) \tau s}\right)}{1-R_{1}(s) R_{2}(s) e^{-2 \tau s}} e^{-\beta \tau s}
$$

The latter form emphasizes the pure delay of the system, which is $\beta \tau$, and is also convenient for the special cases given in Table 1. 
Table 1

Special cases of the transfer function

\begin{tabular}{cc}
\hline Case & $G\left(x, x_{0}, s\right)$ \\
$x_{0}=0$ & $\frac{1}{2 \phi s} \cdot \frac{\left(1+R_{1}(s)\right)\left(1+R_{2}(s) e^{-2(1-\beta) \tau s}\right)}{1-R_{1}(s) R_{2}(s) e^{-2 \tau s}} e^{-\beta \tau s}$ \\
$x_{0}=x$ & $\frac{1}{2 \phi s} \cdot \frac{\left(1+R_{1}(s) e^{-2 \eta \tau s}\right)\left(1+R_{2}(s) e^{-2(1-\eta) \tau s}\right)}{1-R_{1}(s) R_{2}(s) e^{-2 \tau s}}$ \\
$x_{0}=x=0$ & $\frac{1}{2 \phi s} \cdot \frac{\left(1+R_{1}(s)\right)\left(1+R_{2}(s) e^{-2 \tau s}\right)}{1-R_{1}(s) R_{2}(s) e^{-2 \tau s}}$ \\
$x_{0}=0, x=L$ & $\frac{1}{2 \phi s} \cdot \frac{\left(1+R_{1}(s)\right)\left(1+R_{2}(s)\right)}{1-R_{1}(s) R_{2}(s) e^{-2 \tau s}} e^{-\tau s}$ \\
\hline
\end{tabular}

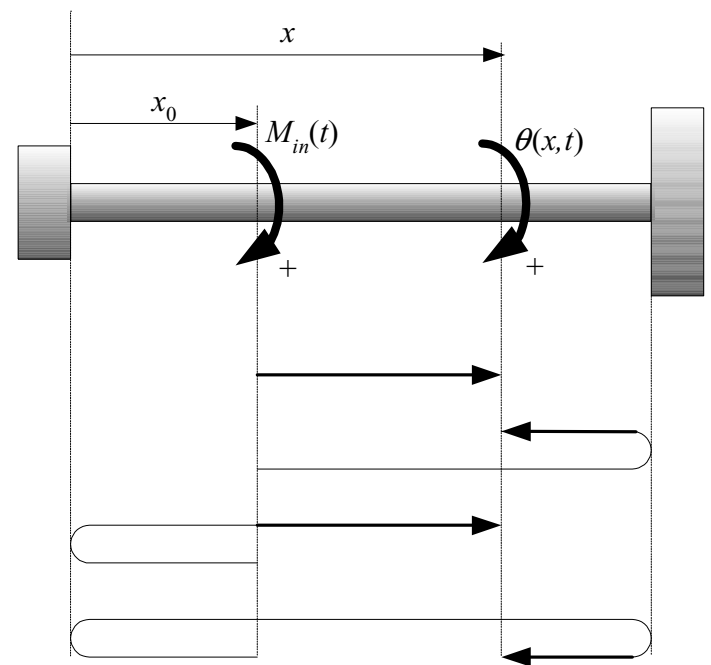

Fig. 2. The four time delays in the numerator of the transfer function in Eq. (8).

The delays in the transfer function can be related to a wave traveling through the shaft. Since $c$ is the wave propagation velocity, $\tau$ is the time required for the wave to travel from one end to another, and can be regarded as the characteristic time of the structure. The four time delays in the numerator of $G\left(x, x_{0}, s\right)$ represent the four possible routes of the wave from $x_{0}$ to $x$, without completing a full cycle, and are shown in Fig. 2 for $x>x_{0}$. The shortest route is clearly the direct one, and it is the pure time delay existing in the system, as can be seen from Eq. (8).

$R_{i}(s)$ are dynamic reflection coefficients of the motion at each end. Some special cases are of particular interest. If the end is free, then $R_{i}(s)=1$ and the wave is returned identically. A fixed end is obtained by $K_{i} \rightarrow \infty$ (equivalently $J_{i} \rightarrow \infty$ ), which results in $R_{i}(s)=-1$. The wave is then returned identically but with an opposite sign. If the end contains no damper, then it follows immediately that $R_{i}(s) R_{i}(-s)=1$ and consequently $\left|R_{i}(j \omega)\right|=1$. This property is an indication of conservation of energy, which exists in systems without dampers.

We conclude this section by noting that a transfer function, that is based on delays, representing the wave motion along the flexible link, and dynamic reflection coefficients, describes the system in terms of the moving wave approach. This is a major departure from the more common modal approach where the moving waves are described as an infinite sum of standing waves.

\section{The absolute vibration suppression (AVS) controller}

The exact and explicit form of the infinite dimension transfer function leads to development of a dedicated controller for the flexible system. It was shown in [5] that any non-collocated controller will destabilize the system, therefore 


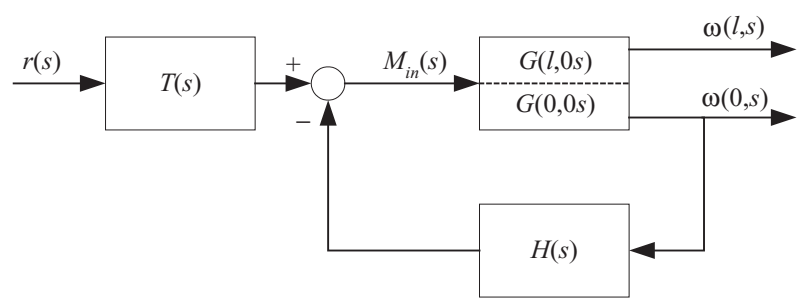

Fig. 3. Velocity control loop.

we consider a collocated control. Furthermore, for reasons that will become clear later, we set the measurement and actuation point at $x=0$, which is the natural choice for any controller. In this paper we focus on velocity control, and the closed loop system is shown in Fig. 3. Notice that while the feedback is closed on $\omega(0, s)$ the performance may be required elsewhere, at $\omega(l, s)$ where typically $l=L$.

The collocated rate feedback control law is given by

$$
M_{i n}(s)=T(s) r(s)-H(s) \cdot \omega(0, s)
$$

The role of $T(s)$ is mainly to ensure zero steady state errors, and it is determined later. Consider the following feedback transfer function

$$
H(s)=\frac{2 \phi R_{1}(s)}{1+R_{1}(s)}
$$

Combining (10) and (8) for $x_{0}=x=0$, hence $\beta=\eta=0$, the closed loop transfer function with the AVS controller is

$$
\begin{aligned}
G_{C L}(x, 0, s) & =\frac{\omega(x, s)}{r(s)}=\frac{T(s) G_{\omega}(x, 0, s)}{1+H(s) G_{\omega}(0,0, s)} \\
& =\frac{\frac{1}{2 \phi} T(s)\left(1+R_{1}(s)\right)\left(1+R_{2}(s) e^{-2(1-\beta) \tau s}\right) e^{-\beta \tau s}}{1-R_{1}(s) R_{2}(s) e^{-2 \tau s}+R_{1}(s)\left(1+R_{2}(s) e^{-2 \tau s}\right)} \\
& =\frac{1}{2 \phi} T(s)\left(1+R_{2}(s) e^{-2(1-\beta) \tau s}\right) e^{-\beta \tau s}
\end{aligned}
$$

$G_{\omega}(x, 0, s)=s G(x, 0, s)$ is the transfer function from the moment to the angular velocity. It is identical with the one in Eq. (8) except for the $s$ in the denominator that is cancelled. It is clear that the selected $H(s)$ eliminates the delay from the closed loop characteristic equation. It can be shown that this is possible only when the actuation is at an end, hence the choice $x=0$. At $x=L$, which is usually the point of interest, this expression becomes

$$
\frac{\omega(L, s)}{r(s)}=\frac{s \cdot T(s)}{J_{2} s^{2}+\left(\phi+D_{2}\right) s+K_{2}} \cdot e^{-\tau s}
$$

Further simplification occurs when the load contains no springs, which is the common case in control systems. With $T(s)=\phi+D_{2}$ the closed loop transfer function becomes a unity gain first order transfer function with a time constant $\mathbf{J}_{2} /\left(\phi+D_{2}\right)$ and the unavoidable delay of one $\tau$.

$$
\frac{\omega(L, s)}{r(s)}=\frac{1}{\frac{J_{2}}{\phi+D_{2}} s+1} e^{-\tau s}
$$

As can be seen from Eqs (11), (12) or (13), the control law has eliminated the infinite number of vibratory poles, hence the name Absolute Vibration Suppression (AVS). The special structure of the AVS controller leads to several observations:

1. The AVS controller is a function of only the shaft properties (material and cross section) and the boundary conditions at the actuation end. More importantly, it is not a function of the shaft length or the boundary conditions at the load end, and is therefore robust for changes in the load. 
2. The closed loop transfer function is only a function of the load and does not depend on the actuator properties.

3. In the typical case of free, or damper only, actuating side boundary conditions the AVS controller becomes a pure gain. .

4. As explained before, the flexible shaft modes are eliminated from the closed loop transfer function. This is achieved by completely absorbing the traveling wave when it returns from the load-side end to the actuation end. Similar motivation lead to the development of the wave-echo controller $[10,13]$.

5. The sum of the AVS controller and the boundary condition equals exactly to the critical damping $\phi$. As a result, the controller matches the shaft impedance at the actuation end and thus no reflection occurs at this end and it becomes a sink.

We conclude this section by considering the actual application of the control law. A detailed expression for the feedback controller is

$$
H(s)=\frac{2 \phi R_{1}(s)}{1+R_{1}(s)}=-J_{1} s+\left(\phi-D_{1}\right)-\frac{K_{1}}{s}
$$

As was already mentioned, in most applications the end does not contain a spring (if it does the problem usually becomes position control), hence the integral terms vanishes. If the inertia cannot be neglected, the AVS controller contains non minimum phase zeros. To avoid inverse response it is positioned in the feedback rather in the forward loop, as in Fig. 3. The controller is actually PD acting on velocity. One way to implement it is by using acceleration measurement.

\section{Stability and robustness}

The stability of the nominal closed loop system is evident from the denominator of the closed loop transfer function (12). A Root Locus analysis (see e.g. [8]) of the system with a controller $k H(s)$ shows that at $k=1$, which is the true AVS controller, all the poles go to $-\infty$, hence disappear. This affects not only stability but performance as well. In cases where the ends do not contain inertia the response is ideally instantaneous, much faster than any other controller that is based on a finite dimension model. In cases where inertia exists, the response is ripple free and has a first order shape. Another advantage is that the design is simple and can be easily automated. Yet another important feature of the AVS controller is that it depends only on local properties, thus completely independent of the load side. The load may change from one task to another, often with strong influence on stability and performance of controllers designed by other methods. In that aspect the robustness of the AVS controller is absolute, both for stability and for wave absorption.

These favorable properties where discussed in previous works, e.g. [5,6,9]. However, since the procedure involves exact matching of terms in the plant model and in the controller, it is important to consider the robustness properties of the AVS controller to other types of uncertainty. This paper looks at the issue of collocation which is central to the method.

A crucial assumption in the AVS development is the sensor-actuator collocation. Assume that the actuation is indeed at $x_{0}=0$, but the measurement is at $x=\beta L$ ( $\beta=0$ in the nominal case). The open loop transfer function is then

$$
H(s) G_{\omega}(\beta, 0, s)=\frac{R_{1}\left(1+R_{2} e^{-2(1-\beta) \tau s}\right) e^{-\beta \tau s}}{1-R_{1} R_{2} e^{-2 \tau s}}
$$

In the analysis that follows we make the plausible assumptions that the system has no springs and that an inertia may exist only at the load end.

Case 1: No inertia at the load end. In this case the reflection coefficients $R_{i}(s)$ are constant and consequently the same holds for $H(s)$.

$$
R_{i}=\frac{\phi-D_{i}}{\phi+D_{i}} \quad, \quad i=1,2 \quad \Rightarrow \quad H(s)=\phi-D_{1}
$$

The open loop transfer function is then given by 

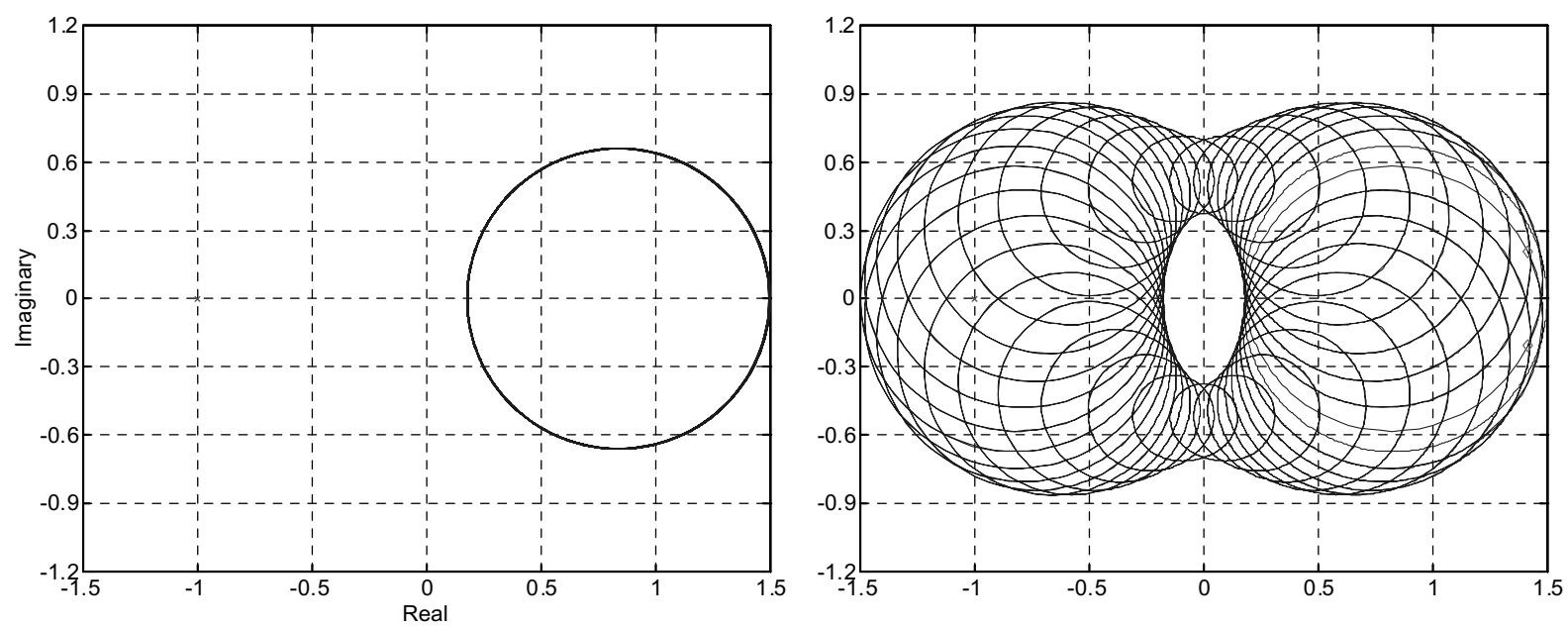

Fig. 4. Nyquist plot of a system with end dampers $\left(D_{1}=D_{2}=\phi / 4\right)$. Left - collocated, right - noncollocated $(\beta=0.05)$.

$$
H(s) G_{\omega}(0,0, s)=\frac{R_{1}\left(1+R_{2} e^{-2 \tau s}\right)}{1-R_{1} R_{2} e^{-2 \tau s}}
$$

For $s=j \omega$ it can be shown that the Nyquist plot of the open loop system in Eq. (17) is the circle, shown in Fig. 4 , with the following center $O$ and radius $R$.

$$
O=R_{1} \frac{1+R_{1}^{2} R_{2}^{2}}{1-R_{1}^{2} R_{2}^{2}} \quad, \quad r=\frac{\left|R_{1} R_{2}\right|\left(1+R_{1}\right)}{1-R_{1}^{2} R_{2}^{2}}
$$

It can further be shown that the closed loop is stable for any damping value since the point $(-1,0)$ is always outside the circle. Notice that the plot repeats itself infinitely since the open loop transfer function is the same for $\omega$ and for $\omega+n \pi / \tau$. Suppose now that the measurement point is not exactly at $x=0$, but at a distance $x=\beta L$ with a small, even infinitesimal, $\beta$. Due to the additional delay, and lack of perfect 'alignment' of the numerator and the denominator, the plot is no longer along a single circle. The Nyquist plot in this case can be looked at as two motions in parallel: A fast one of the point along the circle and a slower one of the circle about the origin. Together they yield the plot in the right hand side of Fig. 4. The plot encircles the point $(-1,0)$ and the closed loop system becomes unstable.

Case 2: the load end has an inertia. In this case the reflection coefficients $R_{i}(s)$ are given by.

$$
R_{1}=\frac{\phi-D_{1}}{\phi+D_{1}}, R_{2}=\frac{-J_{2} s+\phi-D_{2}}{J_{2} s+\phi+D_{2}}
$$

Since the actuation end does not contain an inertia, the controller is still $H(s)=\phi-D_{1}$. Notice that for large $s$ (large $j \omega) R_{i} \rightarrow-1$ and the plot converges to a single circle that repeats itself. The situation is then similar to the previous case. The nominal and non-collocated Nyquist plots are shown in Fig. 5. Again, non-collocation causes instability in this case.

Robust design. The key to the robust design is the observation that the encirclements of the critical point $(-1,0)$ occurs at high frequency. To make the system robust to small non-collocation we suggest adding a low pass filter to the AVS controller so that the amplitude of the Nyquist plot will reduce with frequency. We denote that controller Robust AVS (RAVS). A Nyquist plot of the same systems as in Figs 4 and 5, but with properly tuned filters is shown in Fig. 6. The first crossing of the negative real axis occurs now to the right of the point $(-1,0)$ and the systems are stable. For small non-collocation the cut-off frequency of the filter can be high enough so that the degradation at low frequency and of the time response is minimal. The simple controller design algorithm, for all types of boundary conditions, consists of the following stages:

1. Draw the Nyquist plot of the non-collocated open loop with the nominal AVS controller and find the phase crossover frequency (for which the plot first crosses the negative real axis). 

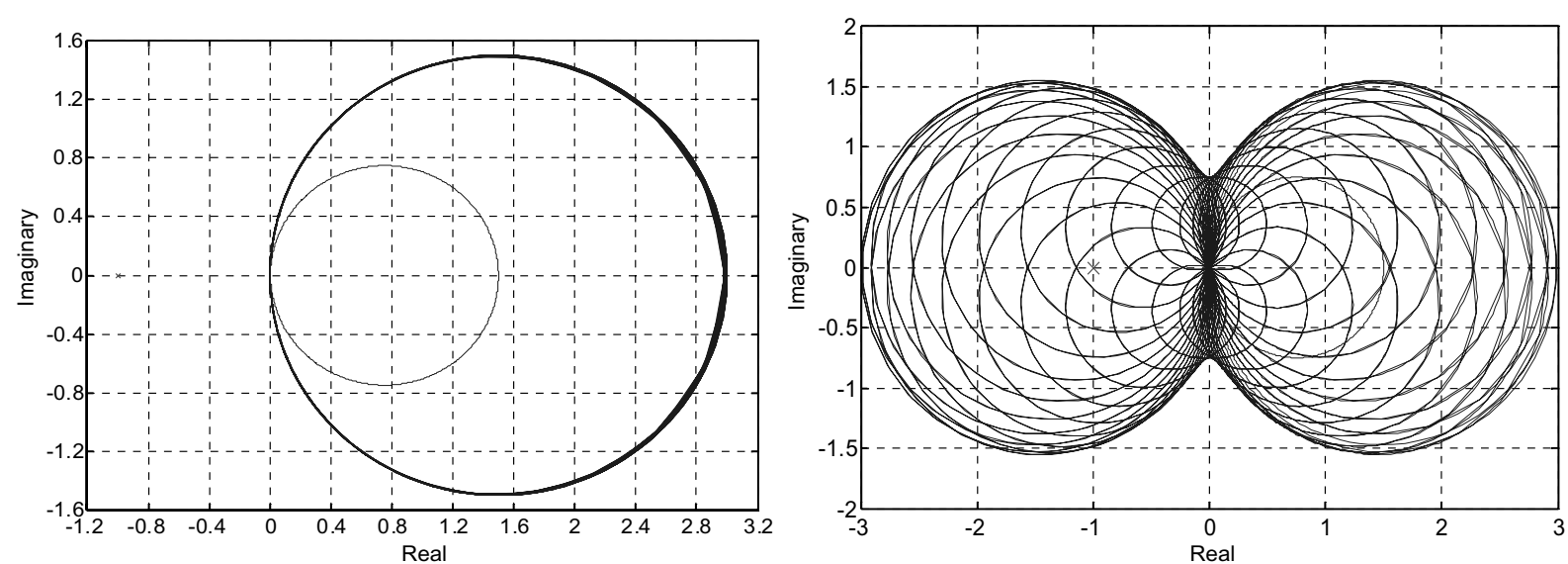

Fig. 5. Nyquist plot of the a system with with end dampers $\left(D_{1}=D_{2}=\phi / 4\right)$ and inertia at the load $\left(J_{2}=20 \phi \tau\right)$. Left - collocated, right noncollocated $(\beta=0.05)$.
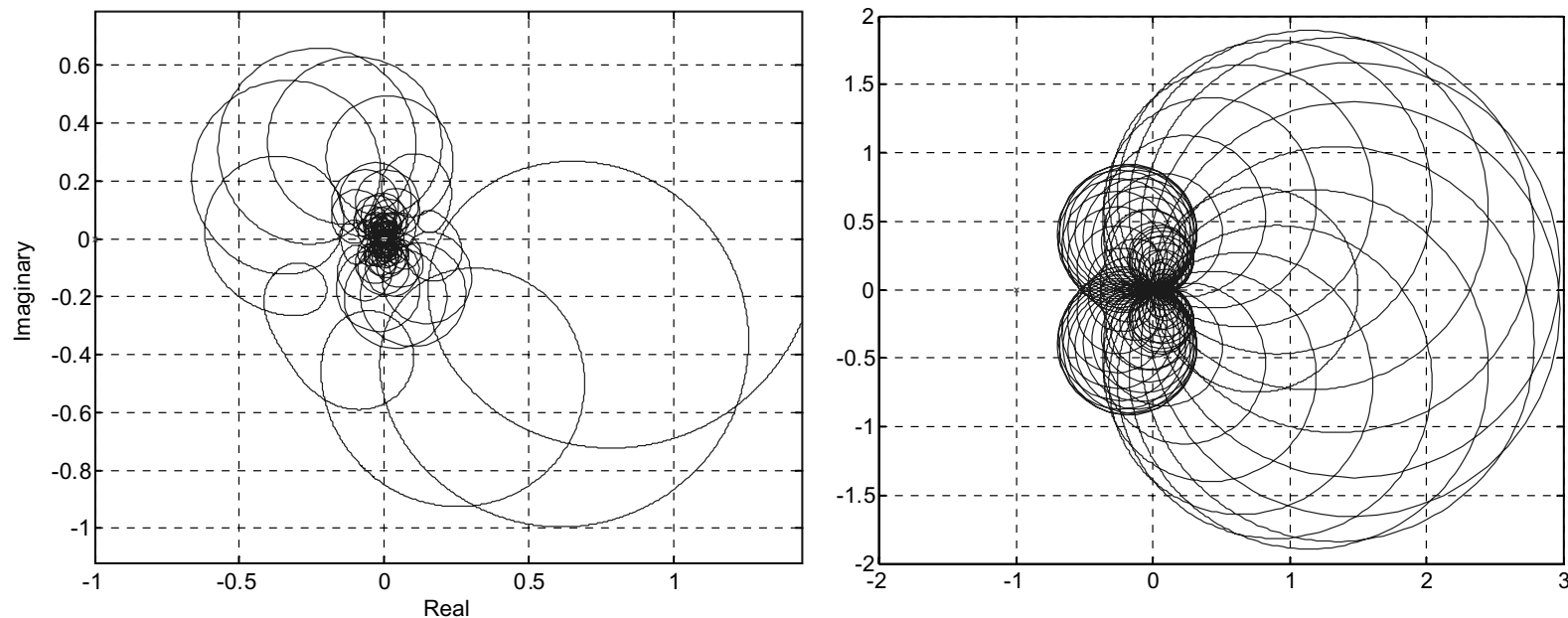

Fig. 6. Nyquist plot of the non-collocated systems 4 with RAVS controller. Left- the system in Fig. 4, right - the system in Fig. 5.

2. If the plot does not encircle the point $(-1,0)$, or not too close to it, the AVS controller can be used as is. Otherwise,

3. Add a low pass filter to the nominal controller. a first guess as to the required cut-off frequency may be the cross over frequency found in step 1.

4. Adjust the filter parameters to obtain the required stability margins. There is no closed form formula for that, but the process is simple and usually only few iterations are required.

From the Nyquist plots in Fig. 6 it is evident that a simple, first order, filter would suffice to stabilize both cases as the amplitude of the closed loop is bounded. The crossover frequency in step 1 is inversely proportional to the amount of non-collocation. For small non-collocation this frequency, which is also roughly the same as the cut-off frequency of the filter, is high, so the performance at low frequencies is only marginally affected.

\section{Conclusion}

The paper considers the Absolute Vibration Suppression (AVS) controller, its properties and some issues regarding its robust implementation. A closed form expression for the accurate, infinite dimension, transfer function of a 
flexible structure is derived and analyzed. The building blocks of the transfer function are time delays, representing the wave motion, and low order rational expressions, representing the boundary phenomena. A fundamental issue is the explicit recognition of the wave motion and reflection as the principal mechanism of the response. This well-known fact is heavily masked, practically absent, in FEM and modal models. With this model, the AVS control law was presented. Its main feature is elimination the infinite number of vibratory poles by means of a collocated rate feedback. A physical explanation is that the controller makes the actuating end a sink for incoming wave, or equivalently, impedance matching. Finally, the stability of the closed loop was investigated using the Nyquist stability criterion. It was shown that even a small non-collocation between the sensor and the actuator may cause instability, and that the robustness can be improved by using RAVS, the robust version of the AVS controller.

\section{References}

[1] M.A. Lau and L.Y. Pao, Input Shaping and Time-Optimal Control of Flexible Structures, Automatica 39(5) (2003), 893-890.

[2] M.J. Balas, Feedback Control of Flexible Systems, IEEE Transactions on Automatic Control 23 (1978), $673-679$.

[3] L. Silverberg, Uniform damping control of spacecraft, Journal of Guidance Control and Dynamics 9 (1986), $221-227$.

[4] V.A. Spector and H. Flashner, Modeling and Design Implications of Noncollocated Control in Flexible Systems, IEEE Transactions on Automatic Control AC-35 (1990), 186-193.

[5] Y. Halevi, Control of flexible structures governed by the wave equation using infinite dimensional transfer functions, ASME Journal of Dynamic Systems, Measurement, and Control 127 (2005), 579-588.

[6] Y. Halevi and C. Wagner-Nachshoni, Transfer Function Modeling of Multi-Link Flexible Structures, Journal of Sound and Vibration 296(1-2) (2006), 73-90.

[7] B. Yang and C.A. Tan, Transfer functions of one dimensional distributed parameter systems, ASME Journal of Applied Mechanics 59 (1991), 1009-1014.

[8] N. Raskin and Y. Halevi, Rate feedback control of free-free uniform flexible rod, Journal of Guidance, Control and Dynamics 24 (2001), 1037-1040.

[9] Y. Halevi and C. Wagner-Nachshoni, Feedback Control of Flexible Structures with Non-Uniform Rods, Proceedings of the American Control Conference, Denver, CO, June 2003, pp. 2646-2651.

[10] I. Peled, Y. Halevi and W.J. O'Connor, Catching the wave - on the relationship between wave based control, absolute vibration suppression and input shaping, ISMA, Luven, Belgium, September 2008.

[11] A.H. von Flotow, Disturbance propagation in structural networks, Journal of Sound and Vibration 106 (1986), $433-450$.

[12] H. Alli and T. Singh, On the Feedback Control of the Wave Equation, Journal of Sound and Vibration 234(4) (2000), 625-640.

[13] W.J. O'Connor, A Gantry Crane Problem Solved, Journal of Dynamic Systems, Measurement and Control 125 (2003), 569-576. 

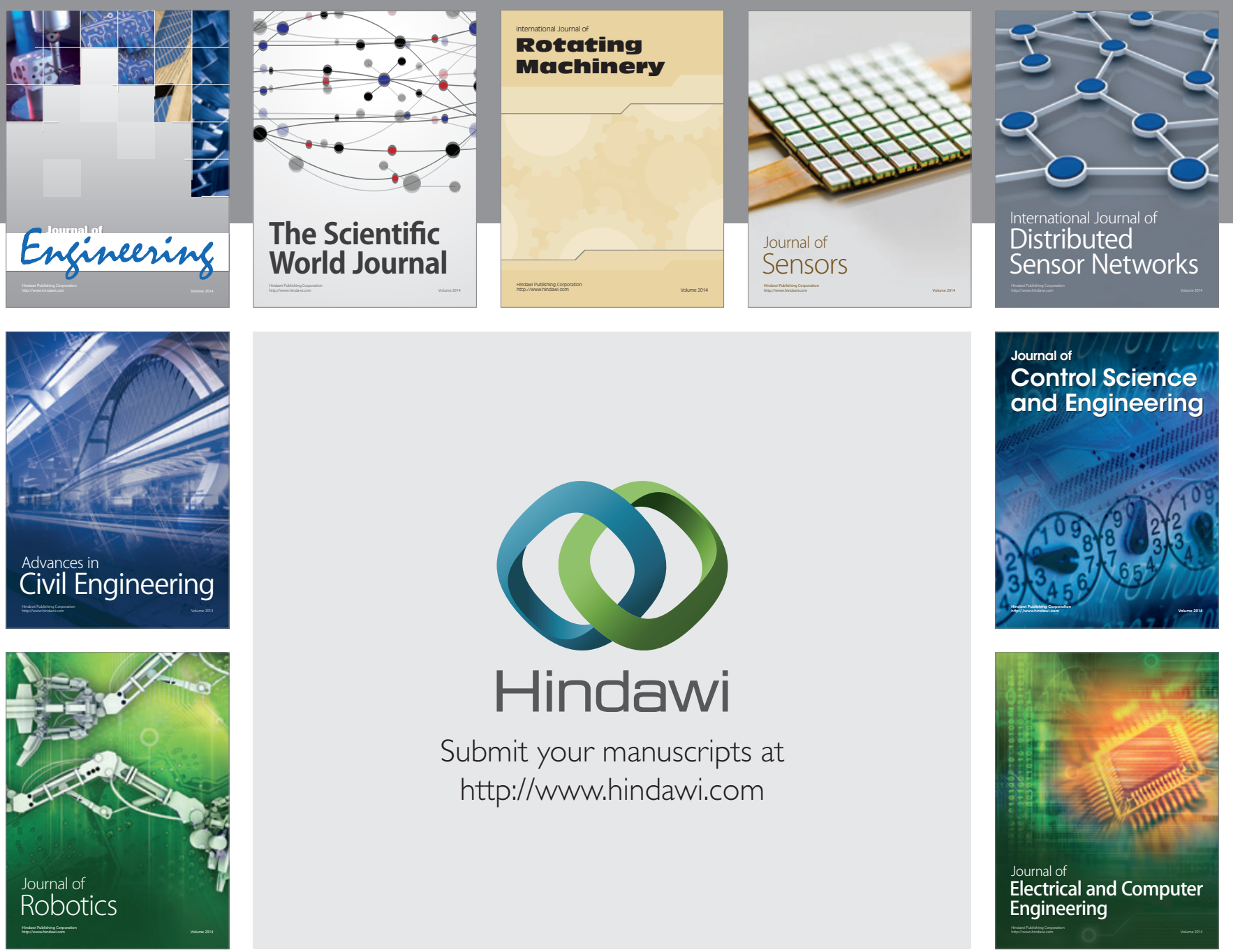

Submit your manuscripts at

http://www.hindawi.com
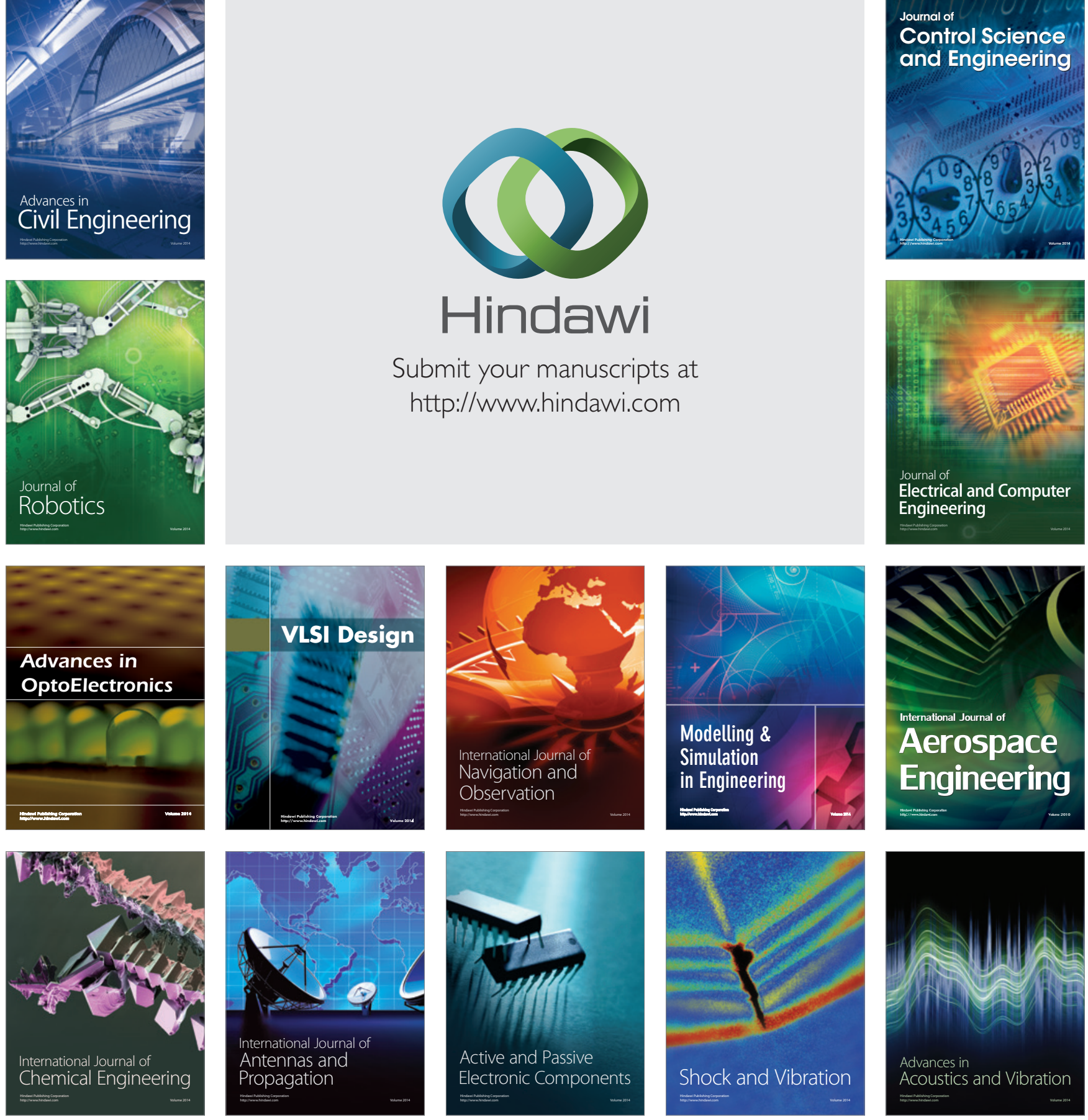